\title{
APPROXIMATION OF AN ALGEBRAIC NUMBER BY PRODUCTS OF RATIONAL NUMBERS AND UNITS
}

\author{
CLAUDE LEVESQUE ${ }^{\bowtie}$ and MICHEL WALDSCHMIDT \\ (Received 11 May 2011; accepted 1 February 2012; first published online 7 February 2013) \\ Communicated by I. E. Shparlinski \\ Dedicated to the memory of Alf van der Poorten
}

\begin{abstract}
We relate a previous result of ours on families of Diophantine equations having only trivial solutions with a result on the approximation of an algebraic number by products of rational numbers and units. We compare this approximation with a Liouville type estimate, and with an estimate arising from a lower bound for a linear combination of logarithms.
\end{abstract}

2010 Mathematics subject classification: primary 11D59; secondary 11J87, 11J68, 11J86, $11 \mathrm{~J} 17$.

Keywords and phrases: Diophantine equations, Diophantine approximation, Liouville inequality, families of Thue-Mahler equations.

\section{Introduction}

In a previous paper [3], we proved that certain families of Diophantine equations have only trivial solutions. In this note (Theorem 3), we show how to deduce from our results on families of Diophantine equations [3] some results on the approximation of an algebraic number by products of rational numbers and units. Since the proofs rest on Schmidt's subspace theorem, these results are noneffective. They improve elementary estimates (Proposition 1) obtained along the lines of Liouville's arguments. A different type of estimate (Theorem 6), which is effective, is achieved by means of a lower bound for a linear combination of logarithms of algebraic numbers.

\section{A variant of the Liouville inequality}

Rational numbers will be written $p / q$ with $q>0$ and $\operatorname{gcd}(p, q)=1$ (and with $q=1$ in case $p=0$ ). When $\alpha$ is an algebraic number of degree $d$, its minimal polynomial will be denoted by

$$
f(X)=a_{0} X^{d}+a_{1} X^{d-1}+\cdots+a_{d} \in \mathbf{Z}[X]
$$

(C) 2013 Australian Mathematical Publishing Association Inc. 1446-7887/2013 \$16.00 
where $a_{0}>0$. In $\mathbf{C}[X]$, this polynomial splits as

$$
f(X)=a_{0} \prod_{\sigma}(X-\sigma(\alpha))
$$

where $\sigma$ in the product runs through the set of embeddings of the field $K:=\mathbf{Q}(\alpha)$ in C. We denote by $|\alpha|$ the maximum complex modulus of the algebraic conjugates of $\alpha$ in $\mathbf{C}$ :

$$
|\alpha|=\max _{\sigma}|\sigma(\alpha)| .
$$

The absolute logarithmic height of $\alpha$ (see [7, Ch. 3]) is

$$
\mathrm{h}(\alpha)=\log a_{0}+\sum_{\sigma} \log \max \{1,|\sigma(\alpha)|\} .
$$

The proof of the next result is close to that of Liouville.

Proposition 1. Let $\alpha \in \mathbf{C}$ be an algebraic number of degree $d$ with minimal polynomial as in (1). Then for any $p / q \in \mathbf{Q}$ and for any unit $\varepsilon$ of $\mathbf{Q}(\alpha)$ such that $\varepsilon \alpha \neq p / q$,

$$
\left|\alpha-\frac{p}{q} \cdot \varepsilon^{-1}\right| \geq \frac{\kappa_{1}}{q^{d}|\varepsilon|^{d-1}|\varepsilon|} \quad \text { with } \kappa_{1}=\frac{1}{a_{0}(2|\alpha|+1)^{d-1}} .
$$

The conclusion can also be written: for any $p / q \in \mathbf{Q}$ and for any unit $\varepsilon$ of $\mathbf{Q}(\alpha)$ such that $\varepsilon \alpha \neq p / q$,

$$
|q \varepsilon \alpha-p| \geq \frac{\kappa_{1}}{(q|\varepsilon|)^{d-1}}
$$

Proposition 1 holds for any algebraic integer $\varepsilon$ lying in $\mathbf{Q}(\alpha)$, not only units, provided that we take into account the leading coefficient of the minimal polynomial of $\varepsilon$. Indeed, from [7, Proposition 3.14] (which follows from the fact that the norm of a nonzero rational integer has absolute value at least 1 and which also follows from the product formula), one deduces that for any algebraic number field $K$ of degree $d$, any element $\gamma$ in $K$ and any rational number $p / q$ with $q \gamma \neq p$,

$$
|q \gamma-p| \geq \frac{1}{(|p|+q)^{d-1} e^{d \mathrm{~h}(\gamma)}} .
$$

We prefer to restrict the situation in Proposition 1 to the special case where $\gamma=\varepsilon \alpha$ for the sake of comparison with Theorems 3 and 6.

Proof. We assume that the rational number $p / q \in \mathbf{Q}$ and the unit $\varepsilon$ of $\mathbf{Q}(\alpha)$ satisfy $\varepsilon \alpha \neq p / q$ and we aim to prove (a third formulation for) the conclusion of Proposition 1, namely

$$
\left|\varepsilon \alpha-\frac{p}{q}\right| \geq \frac{\kappa_{1}}{q^{d} \mid{ }^{d-1}} .
$$

As we have just seen, this will complete the proof of Proposition 1. 
We consider the embeddings $\sigma$ of $\mathbf{Q}(\alpha)$ into $\mathbf{C}$; since $\alpha \in \mathbf{C}$, one of them is the inclusion of $\mathbf{Q}(\alpha)$ in $\mathbf{C}$, which we denote Id. Letting

$$
f_{\varepsilon}(X)=a_{0} \prod_{\sigma}(X-\sigma(\varepsilon \alpha)) \quad \text { and } \quad F_{\varepsilon}(X, Y)=Y^{d} f_{\varepsilon}(X / Y),
$$

we can write

$$
\left|F_{\varepsilon}(p, q)\right|=a_{0} q^{d}\left|\varepsilon \alpha-\frac{p}{q}\right| \prod_{\sigma \neq \mathrm{Id}}\left|\sigma(\varepsilon \alpha)-\frac{p}{q}\right| .
$$

Since $q \geq 1, \kappa_{1}<1$ and $|\varepsilon| \geq 1$, the inequality that we want to establish is trivial if $|\varepsilon \alpha-p / q| \geq 1$. Therefore we can assume that $|\varepsilon \alpha-p / q|<1$, in which case, for every $\sigma$

$$
\left|\sigma(\varepsilon \alpha)-\frac{p}{q}\right| \leq|\sigma(\varepsilon \alpha)-\varepsilon \alpha|+1 \leq 2|\varepsilon \alpha|+1 \leq(2|\alpha|+1)|\varepsilon| .
$$

By assumption $\varepsilon \alpha \neq p / q$; hence, for each $\sigma$, the Galois conjugate $\sigma(\varepsilon \alpha)$ of $\varepsilon \alpha$ is distinct from $\sigma(p / q)=p / q$, and therefore $F_{\varepsilon}(p, q) \neq 0$. Since $F_{\varepsilon}(p, q) \in \mathbf{Z}$, we have $\left|F_{\varepsilon}(p, q)\right| \geq 1$, and the conclusion follows.

Proposition 1 is trivial when $d=1$. In Section 3, we will show that this result is not optimal when $d \geq 3$, in the sense that we can replace $\kappa_{1}$ by an arbitrarily large constant, provided that $q$ is sufficiently large. Consider the case $d=2$. Let $\alpha$ be a quadratic number. The conclusion of Proposition 1 is still trivial if $\alpha$ is not real. So we suppose that $\alpha \in \mathbf{R}$ and we denote by $\epsilon_{0}$ the fundamental unit greater than 1 of the real quadratic field $\mathbf{Q}(\alpha)$. We plan to investigate how close to a best possible one is the lower bound exhibited in Proposition 1. For ease of notation we assume $\varepsilon>0$ without loss of generality. There are two cases: if $\varepsilon>1$, then we write $\varepsilon=\epsilon_{0}^{n}$ with $n>0$; while if $\varepsilon<1$, then we write $\varepsilon=\epsilon_{0}^{-n}$, again with $n>0$. From Proposition 1 we infer, for all $p / q \in \mathbf{Q}$ and all $n>0$, that

$$
\left|\epsilon_{0}^{n} \alpha-\frac{p}{q}\right| \geq \frac{\kappa_{1}}{q^{2} \epsilon_{0}^{n}} \quad \text { and } \quad\left|\epsilon_{0}^{-n} \alpha-\frac{p}{q}\right| \geq \frac{\kappa_{1}}{q^{2} \epsilon_{0}^{n}} .
$$

The next result shows that, infinitely often, these estimates cannot be improved: the dependence on $q$ is sharp in the quadratic case.

Lemma 2. Let $\epsilon_{0}$ be the fundamental unit greater than 1 of the real quadratic field $\mathbf{Q}(\alpha)$. For any $n \geq 0$, with at most one exception, there exist a constant $\kappa_{2}$ and infinitely many rational numbers $p / q$ such that

$$
\left|\epsilon_{0}^{n} \alpha-\frac{p}{q}\right| \leq \frac{\kappa_{2}}{q^{2} \epsilon_{0}^{n}}
$$

and infinitely many rational numbers $p / q$ such that

$$
\left|\epsilon_{0}^{-n} \alpha-\frac{p}{q}\right| \leq \frac{\kappa_{2}}{q^{2} \epsilon_{0}^{n}}
$$

An admissible value for the constant is $\kappa_{2}=\epsilon_{0}^{n} / \sqrt{5}$. 
However this lemma does not give a satisfactory answer to the question of optimality, because $n$ is fixed and $\kappa_{2}$ depends on $n$. In Section 4 we show that the dependence on $|\varepsilon|$ is not sharp in Liouville's estimate, even in the quadratic case.

Proof of Lemma 2. The possible exception is $n$ such that $\epsilon_{0}^{n} \alpha$ or $\epsilon_{0}^{-n} \alpha$ is rational, and the result follows from a theorem of Hurwitz (see, for instance, [4, Ch. 1, Theorem 2F]).

\section{A refinement of Liouville's estimate}

Let $\alpha$ be an algebraic number of degree $d$ over $\mathbf{Q}$ and let $K$ be the field $\mathbf{Q}(\alpha)$. In this section, we plan to prove the following result.

Theorem 3. For any constant $\kappa>0$, the set of pairs $(p / q, \varepsilon) \in \mathbf{Q} \times \mathbf{Z}_{K}^{\times}$such that $[\mathbf{Q}(\varepsilon \alpha): \mathbf{Q}] \geq 3$ and

$$
\left|\varepsilon \alpha-\frac{p}{q}\right| \leq \frac{\kappa}{q^{d}|\varepsilon|^{d-1}}
$$

is finite.

Theorem 3 is trivial when $d=1$ and when $d=2$. For the proof we can suppose that $d \geq 3$. For $\kappa<\kappa_{1}$, inequality (3) is a consequence of Proposition 1 , and the set of exceptional $(p / q, \varepsilon)$ has at most one element. The point is that Theorem 3 holds true for any arbitrarily large constant $\kappa$. The conclusion can also be stated as

$$
(q \mid \varepsilon)^{d-1}\|q \varepsilon\|+\infty \quad \text { as } \max \{q, \mid \varepsilon\} \longrightarrow+\infty .
$$

We twist the minimal polynomial (1) of $\alpha$ by a unit $\varepsilon$ of $K$ by writing

$$
f_{\varepsilon}(X)=a_{0} \prod_{\sigma}(X-\sigma(\varepsilon \alpha)) \quad \text { and } \quad F_{\varepsilon}(X, Y)=Y^{d} f_{\varepsilon}(X / Y) .
$$

Theorem 3 is a corollary of the following theorem whose proof can be found in [3].

Theorem 4. For any integer $k \neq 0$, the set of triples $(x, y, \varepsilon) \in \mathbf{Z}^{2} \times \mathbf{Z}_{K}^{\times}$with $x y \neq 0$ satisfying

$$
[\mathbf{Q}(\varepsilon \alpha): \mathbf{Q}] \geq 3 \text { and } F_{\varepsilon}(x, y)=k
$$

is finite.

Proof of Theorem 3. Let $k>0$ and let $(p / q, \varepsilon) \in \mathbf{Q} \times \mathbf{Z}_{K}^{\times}$satisfy (3). We have $|\varepsilon| \geq 1$. There is no restriction in supposing $q^{d} \geq \kappa$. Consider relation (2). For $\sigma \neq \mathrm{Id}$, we use the upper bound

$$
\left|\sigma(\varepsilon \alpha)-\frac{p}{q}\right| \leq|\varepsilon \alpha-\sigma(\varepsilon \alpha)|+\left|\varepsilon \alpha-\frac{p}{q}\right| \leq|\varepsilon \alpha-\sigma(\varepsilon \alpha)|+1,
$$

which comes from a weak form of (3). Since

$$
|\varepsilon \alpha-\sigma(\varepsilon \alpha)|+1 \leq 2|\varepsilon \alpha|+1 \leq(2|\alpha|+1) \mid \varepsilon,
$$


we deduce, by taking into account (3), that

$$
\left|F_{\varepsilon}(p, q)\right| \leq a_{0} q^{d}\left|\varepsilon \alpha-\frac{p}{q}\right|(2|\alpha|+1)^{d-1}|\varepsilon|^{d-1} \leq a_{0} \kappa(2|\alpha|+1)^{d-1} .
$$

Theorem 4 allows us to conclude that the set of rational numbers $p / q$ which satisfy (3) is finite.

To illustrate Theorem 3, let us make explicit the case of a cubic field whose unit group is of rank one.

Corollary 5. Let $\alpha$ be a real cubic number which has two Galois imaginary conjugates. Let $\epsilon_{0}$ be the real fundamental unit greater than 1 of the cubic field $\mathbf{Q}(\alpha)$. For any constant $\kappa>0$, the set of pairs $(p / q, n) \in \mathbf{Q} \times \mathbf{Z}$ with $n>0$, such that

$$
\left|\alpha-\frac{p}{q \epsilon_{0}^{n}}\right| \leq \frac{\kappa}{q^{3} \epsilon_{0}^{3 n}}
$$

is finite, and the set of pairs $(p / q, n) \in \mathbf{Q} \times \mathbf{Z}$ with $n>0$, such that

$$
\left|\alpha-\frac{p \epsilon_{0}^{n}}{q}\right| \leq \frac{\kappa}{q^{3}}
$$

is finite.

Proof. In Theorem 3, take $\varepsilon=\epsilon_{0}^{n}$ with $|\varepsilon|=\epsilon_{0}^{n}$ for the first part of the statement of Corollary 5, and $\varepsilon=\epsilon_{0}^{-n}$ with $\bar{\varepsilon}=\epsilon_{0}^{n / 2}$ for the second one.

\section{Effective estimates}

A sharp estimate from below for $|\varepsilon \alpha-p / q|$ in terms of $|\varepsilon|$ can be achieved in an effective way by means of a lower bound for linear combinations of logarithms of algebraic numbers.

Theorem 6. Let $K$ be a number field and let $\alpha \in K$. There exists an effectively computable constant $\kappa_{3}>0$ such that, for any unit $\varepsilon \in \mathbf{Z}_{K}^{\times}$and any rational number $p / q$ with $\varepsilon \alpha \neq p / q$,

$$
\left|\varepsilon \alpha-\frac{p}{q}\right| \geq(\log (\mid \varepsilon+2))^{-\kappa_{3} \log \max \{|p|, q, 2\}} .
$$

We will easily deduce Theorem 6 as a consequence of [7, Proposition 9.21], but we can also deduce it from [1, Theorem 4.1] with an explicit value for $\kappa_{4}$. At the same time we notice that Theorem 6 can be generalized to groups of $S$-units of a number field in place of $\mathbf{Z}_{K}^{\times}$, which amounts to replacing $\mathbf{Z}_{K}^{\times}$by any finitely generated subgroup of the multiplicative group of a fixed number field. 
Proposition 7. Let $m$ and $D$ be positive integers. There exists an effectively computable positive number $\kappa_{4}$ depending only on $m$ and $D$ with the following property. Let $\lambda_{1}, \ldots, \lambda_{m}$ be logarithms of algebraic numbers; define $\alpha_{j}=\exp \left(\lambda_{j}\right)(1 \leq j \leq m)$. Assume that the degree of the number field $\mathbf{Q}\left(\alpha_{1}, \ldots, \alpha_{m}\right)$ over $\mathbf{Q}$ is at most $D$. Let $b_{1}, \ldots, b_{m}$ be rational integers, not all of which are zero. Further, let $B, A_{1}, \ldots, A_{m}$ be positive real numbers. Assume that

$$
\log A_{j} \geq \max \left\{\mathrm{h}\left(\alpha_{j}\right),\left|\lambda_{j}\right|, 1\right\} \quad(1 \leq j \leq m)
$$

and

$$
B \geq \max \left\{\left|b_{1}\right|, \ldots,\left|b_{m}\right|, e\right\} .
$$

Assume further that the number

$$
\Lambda:=b_{1} \lambda_{1}+\cdots+b_{m} \lambda_{m}
$$

is nonzero. Then

$$
|\Lambda|>\exp \left\{-\kappa_{4}(\log B)\left(\log A_{1}\right) \cdots\left(\log A_{m}\right)\right\}
$$

The following auxiliary lemma will also be used in the proof of Theorem 6 .

LEMMA 8. Let $K$ be a number field of degree $d=r_{1}+2 r_{2}$ and unit group of rank $r=r_{1}+r_{2}-1$, where $r_{1}$ is the number of real embeddings of $K$ into $\mathbf{R}$ and $r_{2}$ is the number of pairs of nonreal embeddings of $K$ into $\mathbf{C}$. Let $\epsilon_{1}, \ldots, \epsilon_{r}$ be a basis of the torsion-free part of the group of units $\mathbf{Z}_{K}^{\times}$of $K$. Then there is a constant $\kappa_{8}>0$ such that, for any unit $\varepsilon$ of $K$ written as

$$
\varepsilon=\zeta \epsilon_{1}^{b_{1}} \cdots \epsilon_{r}^{b_{r}}
$$

where $\zeta$ is a root of unity in $K$ and $b_{1}, \ldots, b_{r}$ are rational integers,

$$
\max \left\{\left|b_{1}\right|, \ldots,\left|b_{r}\right|\right\} \leq \kappa_{8} \log \varepsilon .
$$

Proof of Lemma 8. Consider the logarithmic embedding $\lambda$ of $K^{\times}$in $\mathbf{R}^{r_{1}+r_{2}}$ given by

$$
\lambda(\alpha)=\left(\log \left|\sigma_{i}(\alpha)\right|\right)_{1 \leq i \leq r_{1}+r_{2}},
$$

where $\sigma_{1}, \ldots, \sigma_{r_{1}}$ are the real embeddings of $K$ into $\mathbf{R}$ and $\sigma_{r_{1}+1}, \ldots, \sigma_{r_{1}+r_{2}}$ are the pairwise nonconjugate nonreal embeddings of $K$ into $\mathbf{C}$. Denote by $\|\cdot\|_{1}$ the sup norm on $\mathbf{R}^{r_{1}+r_{2}}$, so that, for $\alpha \in K^{\times}$,

$$
\|\lambda(\alpha)\|_{1}=\log |\alpha|
$$

The image of $\mathbf{Z}_{K}^{\times}$under $\lambda$ is a lattice in the hyperplane $H$ of equation

$$
x_{1}+\cdots+x_{r_{1}}+2 x_{r_{1}+1}+\cdots+2 x_{r_{1}+r_{2}}=0 .
$$


A basis of $H$ is $\mathcal{B}=\left\{\lambda\left(\epsilon_{1}\right), \ldots, \lambda\left(\epsilon_{r}\right)\right\}$. We denote by $\|\cdot\|_{2}$ the sup norm on $H$ with respect to the basis $\mathcal{B}$. For $\varepsilon \in \mathbf{Z}_{K}^{\times}$written as

$$
\varepsilon=\zeta \epsilon_{1}^{b_{1}} \cdots \epsilon_{r}^{b_{r}}
$$

where $\zeta$ is a root of unity in $K$ and $b_{1}, \ldots, b_{r}$ are rational integers,

$$
\|\lambda(\varepsilon)\|_{2}=\max \left\{\left|b_{1}\right|, \ldots,\left|b_{r}\right|\right\} .
$$

Lemma 8 follows from the equivalence of the norm $\|\cdot\|_{2}$ and the restriction to $H$ of the norm $\|\cdot\|_{1}$. More explicitly, we deduce (5) by looking at the absolute values of the numbers $b_{i}$ obtained via Cramer's formulas for the solutions of the system of linear equations

$$
b_{1} \log \left|\sigma_{j}\left(\epsilon_{1}\right)\right|+\cdots+b_{r} \log \left|\sigma_{j}\left(\epsilon_{r}\right)\right|=\log \left|\sigma_{j}(\varepsilon)\right| \quad\left(j=1, \ldots, r_{1}+r_{2}\right),
$$

which has rank $r$ since the regulator of $K$ does not vanish.

Proof of Theorem 6. The estimate (4) we want to prove is trivial in the case

$$
\left|\varepsilon \alpha-\frac{p}{q}\right| \geq \frac{|p|}{2 q}
$$

hence we may assume that the number $\gamma:=\varepsilon \alpha q / p$ satisfies

$$
0<|\gamma-1|<\frac{1}{2}
$$

and so the principal value $\lambda_{0}$ of the logarithm of $\gamma$ satisfies (see [7, Exercise 1.1.b])

$$
0<\left|\lambda_{0}\right|<2|\gamma-1| \text {. }
$$

Let $\epsilon_{1}, \ldots, \epsilon_{r}$ be a basis of the torsion-free part of the group $\mathbf{Z}_{K}^{\times}$of units of $K$. Write

$$
\varepsilon=\zeta \epsilon_{1}^{b_{1}} \cdots \epsilon_{r}^{b_{r}}
$$

where $\zeta$ is a root of unity in $K$ and $b_{1}, \ldots, b_{r}$ are rational integers. For $1 \leq j \leq r$, select a $\log$ arithm $\log \epsilon_{j}$ of $\epsilon_{j}$, and set

$$
\lambda_{r+1}=\lambda_{0}-b_{1} \log \epsilon_{1}-\cdots-b_{r} \log \epsilon_{r},
$$

so that $e^{\lambda_{r+1}}=\zeta \alpha q / p$. We use Proposition 7 with $m=r+1, \lambda_{j}=\log \epsilon_{j}$ for $1 \leq j \leq r$ and $b_{r+1}=1$. The number $\kappa_{4}$ is a constant depending only on $\alpha$ and $K$, and we may choose for $A_{1}, \ldots, A_{r}$ constants which also depend only on $\alpha$ and $K$. Moreover, for $A_{r+1}$ and $B$, we take

$$
A_{r+1}=\kappa_{5} \max \{|p|, q, 2\} \quad \text { and } B=\kappa_{6} \log (|\varepsilon|+1),
$$

where again $\kappa_{5}$ and $\kappa_{6}$ are constants depending only on $\alpha$ and $K$. The upper bound for $\max \left\{\left|b_{1}\right|, \ldots,\left|b_{r}\right|\right\}$ follows from Lemma 8 . We deduce that there exists a constant $\kappa_{7}$, depending only on $\alpha$ and $K$, such that

$$
\left|\lambda_{0}\right|=\left|b_{1} \log \epsilon_{1}+\cdots+b_{r} \log \epsilon_{r}+\lambda_{r+1}\right| \geq \exp \left\{-\kappa_{7}(\log B) \log \max \{|p|, q, 2\}\right\} .
$$

The result easily follows from (6) and (7). 


\section{Comparison with a result of Corvaja and Zannier}

Denote by $\|\cdot\|$ the distance to the nearest integer: for $x \in \mathbf{R}$,

$$
\|x\|:=\min _{n \in \mathbf{Z}}|x-n| .
$$

Let $\overline{\mathbf{Q}}$ denote the field of complex numbers which are algebraic over $\mathbf{Q}$. Following [2], call a (complex) algebraic number $\xi$ a pseudo-Pisot number if:

(i) $|\xi|>1$ and all its conjugates have (complex) absolute value strictly less than 1;

(ii) $\xi$ has integral trace $\operatorname{Tr}_{\mathbf{Q}(\xi) / \mathbf{Q}}(\xi) \in \mathbf{Z}$.

The main theorem of Corvaja and Zannier [2], whose proof also rests on Schmidt's subspace theorem, can be stated as follows.

THeOREM 9. Let $\Gamma \subset \overline{\mathbf{Q}}^{\times}$be a finitely generated multiplicative group of algebraic numbers, let $\alpha \in \overline{\mathbf{Q}}^{\times}$be a nonzero algebraic number and let $\eta>0$ be fixed. Then there are only finitely many pairs $(q, \varepsilon) \in \mathbf{Z} \times \Gamma$ with $\delta=[\mathbf{Q}(\varepsilon)$ : $\mathbf{Q}]$ such that $|\alpha q \varepsilon|>1, \alpha q \varepsilon$ is not a pseudo-Pisot number and

$$
0<\|\alpha q \varepsilon\|<\frac{1}{e^{\eta \mathrm{h}(\varepsilon)} q^{\delta+\eta}} .
$$

The special case $\varepsilon=1, \delta=1$ of Theorem 9 is a Roth-type estimate. The proof we gave in Section 3 relies on our result on Diophantine equations in [3], which is a consequence of Schmidt's subspace theorem, while the proof of Corvaja and Zannier in [2] uses Schmidt's fundamental result on linear forms in algebraic numbers directly. It is likely that an improvement of our result could be achieved by adapting the arguments of [2], so one would expect to obtain a refinement of our conclusion which would also include the statement of Theorem 9.

However, it turns out that in some very particular cases, Theorem 9 is weaker than Liouville's estimate (Proposition 1), hence weaker than our Theorem 3. Here is an example. Assume in Theorem 9 that $\Gamma$ is the group of units $\mathbf{Z}_{K}^{\times}$of a number field $K$ of degree $d=\delta$ and that $\alpha \in K$. In this special case, for $\varepsilon \in \mathbf{Z}_{K}^{\times}$, we may replace $\log |\varepsilon|$ by $\mathrm{h}(\varepsilon)$ without spoiling the result, since

$$
\log |\varepsilon| \leq \mathrm{h}(\varepsilon) \leq d \log \mid \varepsilon .
$$

Hence Theorem 9 implies that for any $\eta>0$, there are only finitely many pairs $(q, \varepsilon) \in \mathbf{Z} \times \mathbf{Z}_{K}^{\times}$such that $|\alpha q \varepsilon|>1, \alpha q \varepsilon$ is not a pseudo-Pisot number and

$$
0<\|\alpha q \varepsilon\|<\frac{1}{|\bar{\varepsilon}|^{\eta} q^{d+\eta}} .
$$

In other words, if $|\alpha q \varepsilon|>1$ and $\alpha q \varepsilon$ is not a pseudo-Pisot number, then for all pairs $(q, \varepsilon) \in \mathbf{Z} \times \mathbf{Z}_{K}^{\times}$, except for finitely many of them,

$$
\|\alpha q \varepsilon\| \geq \frac{1}{|\bar{\varepsilon}|^{\eta} q^{d+\eta}} .
$$


It may be observed that a more concise form of this statement is

$$
\lim \inf \frac{d \log q+\log \|\alpha q \varepsilon\|}{\log q+\log |\varepsilon|} \geq 0 \quad \text { as } \max \{q, \mid \varepsilon\} \rightarrow+\infty,
$$

where $(q, \varepsilon) \in \mathbf{Z} \times \mathbf{Z}_{K}^{\times}$with $|\alpha q \varepsilon|>1$ and $\alpha q \varepsilon$ being not a pseudo-Pisot number.

In the case where the pairs $(q, \varepsilon)$ belong to a set in which $|\varepsilon|^{d-1} q^{-1}$ is bounded from above, (9) is weaker than the lower bound

$$
(q \bar{\varepsilon})^{d-1}\|q \alpha \varepsilon\| \geq \kappa_{1}
$$

given by Liouville's inequality (Proposition 1), hence it is weaker than the result which one deduces from Theorem 3.

For the comparison with (4), let us consider a set of pairs $(q, \varepsilon)$ in which $(\log |\varepsilon|)(\log q)^{-1}$ is bounded from above by a positive constant and at the same time $\log \mid \varepsilon(\log q)^{-1}(\log \log q)^{-1}$ is bounded from below by a positive constant. In this case one deduces from Theorem 6 that

$$
\|\alpha q \varepsilon\| \geq \exp \left\{-\kappa_{5}(\log \log (\mid \varepsilon+2)) \log \max \{|q \varepsilon|, 2\}\right\}
$$

where $\kappa_{5}$ is an effectively computable constant depending only on $\alpha$ and $K$. Hence in this special case, the lower bound for $\|\alpha q \varepsilon\|$ which we deduce from (4) is a power of $\log (\mid \varepsilon)$, while Theorem 9 yields a weaker lower bound, namely a power of $\varepsilon$. Thus Theorem 3 sometimes yields sharper estimates than Theorems 6 and 9 when $q$ is large, Theorem 6 is effective and may be sharper than Theorems 3 and 9 when $|\varepsilon|$ is large, while Theorem 9 is most often sharper than Theorems 3 and 6 for an intermediate range. However, we emphasize the fact that Theorem 9 has a wider scope, even if it sometimes happens to be less strong than other results.

We conclude with two selected examples in which we take $\alpha=1$.

ExAmple 10. Consider a cubic number field $K$ with group of units of rank one and let $\epsilon_{0}>1$ be the fundamental unit, so that $\mathbf{Z}_{K}^{\times}=\{1,-1\} \times\left\langle\epsilon_{0}\right\rangle$ and $\epsilon_{0}=\epsilon_{0}$. Theorem 3 states that for any $\kappa>0$, there are only finitely many $(n, q) \in \mathbf{N}^{2}$ such that

$$
\left\|q \epsilon_{0}^{n}\right\| \leq \frac{\kappa}{\epsilon_{0}^{2 n} q^{2}}
$$

This means that the function

$$
(q, n) \longmapsto \epsilon_{0}^{2 n} q^{2}\left\|q \epsilon_{0}^{n}\right\|
$$

tends to infinity as $\max \{q, n\}$ tends to infinity. Liouville's inequality (Proposition 1 ) gives only a lower bound for $\epsilon_{0}^{2 n} q^{2}\left\|q \epsilon_{0}^{n}\right\|$ with an explicit positive constant. The equality (8) cannot be used because $q \epsilon_{0}^{n}$ is a pseudo-Pisot number. The conclusion of Theorem 6 is

$$
\left\|q \epsilon_{0}^{n}\right\| \leq n^{-\kappa_{6} n} q^{-\kappa_{6} \log n}
$$

for $n \geq 2$, which is weaker than the estimates that we deduced from Theorem 3 . 
For an explicit example, let $D$ be an integer greater than 1 and let $\omega=\sqrt[3]{D^{3}-1}>1$. The fundamental unit greater than 1 of the cubic field $\mathbf{Q}(\omega)$ is $\epsilon_{0}=1 /(D-\omega)=$ $D^{2}+D \omega+\omega^{2}$ (see [6]).

ExAmple 11. Let $K$ be a number field of degree $d$. Assume that there are two independent real units $\epsilon_{2}>\epsilon_{1}>1$ in $K$. Since $\epsilon_{1}, \epsilon_{2}$ are multiplicatively independent, the numbers $\log \epsilon_{1}, \log \epsilon_{2}$ are linearly independent over $\mathbf{Q}$, hence $\mathbf{Z} \log \epsilon_{1}+\mathbf{Z} \log \epsilon_{2}$ is a dense subgroup of $\mathbf{R}$ and therefore the multiplicative subgroup of $\mathbf{R}_{+}^{\times}$generated by $\epsilon_{1}$, $\epsilon_{2}$ is dense. Hence there exists a sequence of units $\varepsilon_{n}=\epsilon_{1}^{a_{n}} \epsilon_{2}^{-b_{n}}$ such that $1 / 2 \leq \varepsilon_{n} \leq 2$. The numbers $a_{n}$ and $b_{n}$ are positive integers which tend to infinity. Since

$$
\left|\log \varepsilon_{n}\right|=\left|a_{n} \log \epsilon_{1}-b_{n} \log \epsilon_{2}\right| \leq \log 2,
$$

the limit of the sequence $a_{n} / b_{n}$ is $\left(\log \epsilon_{2}\right) /\left(\log \epsilon_{1}\right)$. For instance, one can take for $a_{n} / b_{n}$ the convergents of the continued fraction expansion of $\left(\log \epsilon_{2}\right) /\left(\log \epsilon_{1}\right)$. The sequences

$$
\left(\frac{\log \sqrt{\varepsilon_{n}} \mid}{a_{n} \log \epsilon_{1}}\right)_{n \geq 1} \text { and }\left(\frac{\log \sqrt{\varepsilon_{n}} \mid}{b_{n} \log \epsilon_{2}}\right)_{n \geq 1}
$$

converge to the positive limit

$$
\max _{\sigma: K \rightarrow \mathbf{C}}\left(\frac{\log \left|\sigma \epsilon_{1}\right|}{\log \epsilon_{1}}-\frac{\log \left|\sigma \epsilon_{2}\right|}{\log \epsilon_{2}}\right) .
$$

For $n$ sufficiently large, we have $\varepsilon_{n} \geq e^{e}$. Liouville's inequality from Proposition 1 is

$$
\left(q \overline{\varepsilon_{n}}\right)^{d-1}\left\|q \varepsilon_{n}\right\| \geq \kappa_{1},
$$

and Theorem 3 yields

$$
\left(q \mid \varepsilon_{n}\right)^{d-1}\left\|q \varepsilon_{n}\right\| \longrightarrow+\infty \quad \text { as } \max \{q, n\} \longrightarrow+\infty
$$

while Theorem 6 gives the lower bound

$$
\left\|q \varepsilon_{n}\right\| \geq \exp \left\{-\kappa_{7}\left(\log \log \overline{\varepsilon_{n}}\right) \log \max \{q, 2\}\right\}
$$

and Theorem 9 yields

$$
\lim \inf \frac{d \log q+\log \left\|q \varepsilon_{n}\right\|}{\log q+\log \left|\varepsilon_{n}\right|} \geq 0
$$

as $\max \{q, n\} \longrightarrow+\infty$ with $q \varepsilon_{n}$ being not a pseudo-Pisot number. Hence Theorem 6 is sharper, when $n$ is large, than the estimate which one deduces from Theorem 3 and than the estimate which one deduces from Theorem 9. 
For an explicit example, let $D$ be an integer greater than 1 and let $\omega=\sqrt[4]{D^{4}-1}>1$. A pair of independent units of the biquadratic number field $\mathbf{Q}(\omega)$ is (see [5])

$$
\epsilon_{1}=D^{2}+\omega^{2}=\frac{1}{D^{2}-\omega^{2}} \quad \text { and } \quad \epsilon_{2}=\frac{1}{D-\omega}=D^{3}+D^{2} \omega+D \omega^{2}+\omega^{3} .
$$

\section{Acknowledgements}

The authors are grateful to Francesco Amoroso, Kálmán Győry and Yann Bugeaud for their remarks on a preliminary version of this paper.

\section{References}

[1] A. Bérczes, J.-H. Evertse and K. Győry, 'Effective results for linear equations in two unknowns from a multiplicative division group', Acta Arith. 136 (2009), 331-349.

[2] P. Corvaja and U. Zannier, 'On the rational approximations to the powers of an algebraic number: solution of two problems of Mahler and Mendès France', Acta Math. 193 (2004), 175-191.

[3] C. Levesque and M. Waldschmidt, 'Familles d'équations de Thue-Mahler n'ayant que des solutions triviales', Acta Arith. 155 (2012), 117-138.

[4] W. M. Schmidt, Diophantine Approximation, Lecture Notes in Mathematics, 785 (Springer, Berlin, 1980).

[5] H.-J. Stender, 'Grundeinheiten für einige unendliche Klassen reiner biquadratischer Zahlkörper mit einer Anwendung auf die diophantische Gleichung $x^{4}-a y^{4}= \pm c(c=1,2,4 \text { oder } 8)^{\prime}, J$. reine angew. Math. 264 (1973), 207-220.

[6] H.-J. Stender, 'Lösbare Gleichungen $a x^{n}-b y^{n}=c$ und Grundeinheiten für einige algebraische Zahlkörper vom Grade $n=3,4,6$ ', J. reine angew. Math. 290 (1977), 24-62.

[7] M. Waldschmidt, Diophantine Approximation on Linear Algebraic Groups, Grundlehren der Mathematischen Wissenschaften, 326 (Springer, Berlin, 2000).

CLAUDE LEVESQUE, Département de Mathématiques et de Statistique,

Université Laval, Québec (Québec), Canada G1V 0A6

e-mail: Claude.Levesque@mat.ulaval.ca

MICHEL WALDSCHMIDT, Institut de Mathématiques de Jussieu, Université Pierre et Marie Curie (Paris 6), 4 Place Jussieu, F-75252 Paris Cedex 05, France

e-mail: miw@math.jussieu.fr 